\title{
Assessing obesity, body fatness and dietary behaviors among adult college students in Hail, Saudi Arabia
}

\author{
"Mo'ez Al-Islam" Faris ${ }^{1,2}$, Suneetha Epuru ${ }^{1,}$ ", Dima Abu-Jamous ${ }^{1}$, Majdi Smadi ${ }^{1}$, Ala'a Eideh ${ }^{1}$, \\ Eyad Alshammari ${ }^{1}$
}

${ }^{1}$ Dept. of Clinical Nutrition, College of Applied Medical Sciences, University of Hail, Hail, Saudi Arabia

${ }^{2}$ Department of Nutrition, Faculty of Pharmacy and Medical Sciences, Petra University, Amman, Jordan

\section{Email address:}

sunny11sai@gmail.com (S. Epuru)

\section{To cite this article:}

"Mo'ez Al-Islam" Faris, Suneetha Epuru, Dima Abu-Jamous, Majdi Smadi, Ala'a Eideh, Eyad Alshammari. Assessing Obesity, Body Fatness and Dietary Behaviors among Adult College Students in Hail, Saudi Arabia. International Journal of Nutrition and Food Sciences. Vol. 3, No. 2, 2014, pp. 60-68. doi: 10.11648/j.ijnfs.20140302.17

\begin{abstract}
The present study objective was to measure the prevalence of obesity and assess body fatness among college students in Hail, Northern Saudi Arabia, and to explore associated dietary behaviors. A sample of 314 adult college students (236 females and 78 males) was recruited, and the prevalence of obesity was measured by assessing body composition using bioelectrical impendence technique. Dietary behaviors were examined to elaborate their relevance to obesity by using a selfadministered questionnaire. Study results revealed that $25.6 \%$ and $14.4 \%$ of the subjects were overweight and obese, respectively. Females were at increased risk for both high risk waist-hip ratio (WHR) $(\mathrm{p}=0.000)$ and high body fat percent $(\mathrm{BF} \%)(\mathrm{p}=0.004)$. Dietary behaviors varied across gender and BMI groups, with males preferring dining out, eating fast foods, and carbonated beverages as compared to females who preferred dining with family, snacking on potato chips, chocolates, cakes, sweets and drank more caffeinated beverages. Both genders were at risk for dietary behaviors like eating less fruits and vegetables. Snacking was inversely associated with overweight and obesity $(p=0.05)$ while drinking caffeinated beverages was positively linked $(\mathrm{p}=0.043)$. Skipping breakfast $(\mathrm{p}=0.006)$, low consumption of fruits $(\mathrm{p}=0.012)$, and frequent restaurant visits $(\mathrm{p}=0.027)$ were significantly associated with prevalence of high $\mathrm{BF} \%$. Results from this study highlight the importance of early identification and correction of the unhealthy dietary and lifestyle behaviors contributing to the high prevalence of obesity in young adults.
\end{abstract}

Keywords: BMI, Dietary Behaviours, Obesity, Percent Body Fat, Young Adults

\section{Introduction}

The Kingdom of Saudi Arabia (KSA) is one among the few countries with the advantage of demographic dividend because of its overwhelmingly young population. Around $29.4 \%$ of the Saudi population is under 14 years old while majority population is below the age of 30 years [1].

During the past decades, KSA has experienced dramatic socioeconomic development accompanied by increased urbanization. Living standards are on a rise, leading to complex changes in patterns of health, disease and mortality by substituting chronic diseases for infectious and communicable diseases as the primary causes of morbidity and mortality [2, 3]. Concurrent with this epidemiological transition trend, the prevalence rates of overweight and obesity are sharply rising in KSA [4]. The available literature indicates that obesity is emerging as a major health problem with approximately three quarters of females and nearly two-thirds of males of adult population in the Kingdom being either overweight or obese [5]. Obesity rates are also alarmingly increasing in the younger populations of $\mathrm{KSA}[4,6]$. Interestingly, studies also indicate variation in the prevalence rates of overweight and obesity in different provinces of Saudi Arabia indicating the disparity in health transition in these regions $[4,6]$.

Hail region in the North of Kingdom was reported to be having the highest percentage of obese population (33.9\%) among all the regions of the KSA [4]. The recent massive development of Hail into a fast growing sub-urban city from a traditional agrarian society emphasizes the pressing need for a thorough epidemiological survey in this region introspecting for lifestyle factors responsible for being epitome of obesity as compared to other regions. This exploration can help in identifying health risk behaviors 
which are specific to Hail region, and will subsequently be advantageous in expanding health awareness and creating public education programs.

Young adults represent a period of various important transitions relevant to later life and health with explicit lifestyle priority issues, calling for specific strategies and approaches. Research suggests greatest increases in obesity occur in individuals between the ages of 18 to 29 years, during the transition from adolescence to adulthood [2] and young women notably having higher prevalence of overweight and obesity as compared to males [7, 8]. Young adults are potentially at risk for poor dietary choices largely because of their very high nutrient demands coupled with incompatibility of their range of preferred foods and patterns of eating. With appetite drives high in order to meet physiological demands, they are strongly influenced by feelings of hunger and select foods based on hedonistic factors [9]. Young adults also tend to feel constrained in terms of time, as they have to integrate their heavy workloads at college, busy social programs and sporting activities which can promote preferences for convenience foods [9].

In view of the foregoing observations, the present investigation was undertaken to assess the prevalence of overweight and obesity along with body composition analysis in a sample of male and female students from the University of Hail [UOH) in Hail City, KSA, and to examine its relevance to their present gender specific dietary behaviors.

\section{Methodology}

\subsection{Study Design, Sample and Data Collection}

A cross sectional survey was planned and conducted in both male and female campuses of University of Hail, Hail, KSA during Nov., 2011 to Jan., 2012. Approximately, a random sample size of 314 students (236 females from female campus representing both Science and Humanities Colleges and 78 males from male campus representing only Science colleges because of logistic reasons) participated in the survey. All enrolled participants were briefed about the purpose of the study and were required to provide a written informed consent before participating in the study. The study protocol was approved by University of Hail Committee on Human Research Ethics. After self reported questionnaires were filled by the participants, trained nutrition students helped them to undergo bioelectric impedance analysis for anthropometric measurements in the standard procedure protecting student privacy by allowing for anonymous and voluntary participation. Pregnant female students were excluded from participation in the study.

\subsection{Anthropometry}

Anthropometric measurements such as body weight and height were measured using standard techniques in the nutrition clinic. Body height was measured using measuring rod (DETECTO, USA) to the nearest $0.01 \mathrm{~m}$, and body weight was measured with the subjects wearing light clothing and no shoes using mechanical flat scale (DETECTO, USA) to the nearest $0.1 \mathrm{~kg}$. Body mass index (BMI) was calculated as the ratio of weight (kilograms) to the square of height (meters). According to World Health Organization (WHO) [10] weight status was classified into four categories: underweight $(\mathrm{BMI}<18.5)$, normal weight (BMI between 18.5 - 24.99), overweight (BMI between 25.00-29.99), and obese (BMI $\geqslant 30.00)$. Body fat percent $(\mathrm{BF} \%)$, skeletal muscle mass, waist to hip ratio (WHR) and other body composition measurements were measured with the help of bioelectric impedance analysis (InBody 520, GE Health Care, USA). Manufacturer's instructions were followed strictly for accurate measurement with InBody 520. WHR was used to identify the study population into low and high risk groups (cut-off for males $\geqslant 0.90$ and for females $\geqslant 0.85$ ) [11]. Normal ranges for $\mathrm{BF} \%$ were considered as follows: $10-20 \%$ for males and $18-28 \%$ for females [12].

\subsection{Assessment Tool}

The questionnaire requested information on socio-demographic and anthropometric information which included age, gender, college, self-reported height and weight followed by questions related to study objectives as follows:

Dietary assessment: Subject's eating patterns and dietary behaviors were assessed by asking how often dietary behaviors like eating fruits, vegetables, breakfast, lunch, dinner, fast foods as well as frequency related to snack food consumption patterns were practiced over the past one month. The questionnaire was adopted from a previously published study [13] where authors have standardized its use among university students. A slight modification was done on the questionnaire to be more suitable with the dietary and food patterns of the Saudi students.

Validation of the Questionnaire: For face and content validity, the questionnaire was initially translated into Arabic and then pre-tested for question accuracy and clarity. Moreover, majority questions in the questionnaire were taken from previously validated instrument [13], where authors have standardized its use among university students.

\subsection{Statistical Analysis}

The data set was cleaned and edited for inconsistencies. Missing data were not statistically computed. Statistical analyses were performed using the Statistical Package for Social Sciences (version 16.0, SPSS, Inc) software. Descriptive statistics such as means and standard deviations were calculated for the continuous variables and frequencies for qualitative data. Analyses of variance (ANOVA), student's t-test and chi-square analysis were used to examine differentials in variables. Results were expressed as mean \pm 
SD. All reported $p$ values were 2-sided and differences were considered statistically significant at $\mathrm{p}<0.05$.

\section{Results}

A total of 313 subjects (18- 24 years, $20.61 \pm 1.56$ years) of which 78 male $(24.9 \% ; 20.97 \pm 1.52$ years $)$ and 235 female $(75.1 \% ; 20.49 \pm 1.55$ years) subjects participated in the present survey. Vast majority of the study subjects were unmarried (90.7 \%); and were from science colleges $(81.5 \%)$. The socioeconomic characteristics of these subjects are reported in Table 1.

Table 1. Socio-demographic characteristics of the study population, $n$ (\%)

\begin{tabular}{lllll}
\hline Characteristics & & Total & Males & Females \\
\hline Sample Size & & $313(100)$ & $78(24.9)$ & $235(75.1)$ \\
& $18-20$ & $167(53.4)$ & $33(42.3)$ & $134(57.0)$ \\
Age Groups (year) & $21-23$ & $133(42.5)$ & $40(51.3)$ & $93(39.6)$ \\
& $\geq 24$ years & $13(4.2)$ & $5(6.4)$ & $8(3.4)$ \\
\multirow{2}{*}{ College } & Humanity & $57(18.5)$ & 0.0 & $57(24.8)$ \\
& Science & $251(81.5)$ & $78(100)$ & $173(75.2)$ \\
Marital Status & Unmarried & $284(90.7)$ & $73(93.6)$ & $211(89.8)$ \\
\hline
\end{tabular}

Table 2 presents anthropometric measurements of the study population. Results imply that, except for BMI and WHR, all other anthropometric variables and body composition components were significantly $(\mathrm{P}<0.05)$ higher in males subjects than in females. For both males as well as females, means of BMI $(24.47 \pm 6.30$ and $24.31 \pm 5.47$, respectively) were closer towards the upper cut-off value of the normal range (BMI: 18.5 to 24.99), while both mean WHR $(0.87 \pm 0.07$ and $0.86 \pm 0.07$, respectively) and mean $\mathrm{BF} \%(24.03 \pm 10.4$ and $36.41 \pm 9.44$, respectively) were well above the normal ranges, auguring for the fact that both genders are equally at risk for developing overweight and obesity in the near future.

Table 2. Anthropometric measurements for the study population (Mean \pm SD)

\begin{tabular}{|c|c|c|c|c|c|}
\hline Measurement & Total & Males & Females & t-test Value (Males vs. Females) & $t$-test Sig. \\
\hline Height $(\mathrm{cm})$ & $160.74 \pm 8.51$ & $172.12 \pm 5.52$ & $156.97 \pm 5.41$ & 21.12 & $0.000 *$ \\
\hline Weight (kg) & $62.95 \pm 16.14$ & $72.63 \pm 18.84$ & $59.74 \pm 13.75$ & 6.50 & $0.000 *$ \\
\hline $\operatorname{BMI}\left(\mathrm{kg} / \mathrm{m}^{2}\right)$ & $24.35 \pm 5.67$ & $24.47 \pm 6.30$ & $24.31 \pm 5.47$ & 0.20 & 0.840 \\
\hline WHR & $0.87 \pm 0.07$ & $0.87 \pm 0.06$ & $0.86 \pm 0.07$ & 1.40 & 0.164 \\
\hline $\mathrm{BFM}(\mathrm{kg})$ & $21.91 \pm 11.52$ & $19.26 \pm 13.87$ & $22.79 \pm 10.53$ & 2.06 & $0.042 *$ \\
\hline $\mathrm{BF} \%$ & $33.33 \pm 11.06$ & $24.03 \pm 10.4$ & $36.41 \pm 9.44$ & 9.32 & $0.000 *$ \\
\hline
\end{tabular}

*Significant difference at $\mathrm{P}<0.05$.

Table 3. Obesity and WHR in the study population.n (\%)

\begin{tabular}{|c|c|c|c|c|}
\hline Category & $\operatorname{Total}(n=313)$ & Males $(n=78)$ & Females $(n=235)$ & $x^{2}$ Value \\
\hline Underweight & $38(12.1)$ & $12(15.4)$ & $26(11.1)$ & \multirow{4}{*}{$1.963 \quad(P=0.583)$} \\
\hline Normal BMI & $150(47.9)$ & $33(42.3)$ & $117(49.8)$ & \\
\hline Overweight & $80(25.6)$ & $20(25.6)$ & $60(25.5)$ & \\
\hline Obese & $45(14.4)$ & $13(16.7)$ & $32(13.6)$ & \\
\hline Low risk WHR group (Males $\leq 0.89$ and females $\leq 0.84$ ) & $159(50.80)$ & $53(67.9)$ & $106(45.1)$ & \multirow{2}{*}{$12.226 \quad(P=0.0001) *$} \\
\hline High Risk WHR group (Males $\geq 0.90$ and females $\geq 0.85$ ) & $154(49.2)$ & $25(32.1)$ & $129(54.9)$ & \\
\hline Low $\mathrm{BF} \%$ ( $\leq 9$ for males and $\leq 17$ for females $)$ & $11(3.51)$ & $4(5.1)$ & $7(3.0)$ & \multirow{3}{*}{$11.051 \quad(P=0.004) *$} \\
\hline Normal BF \% (10-20 for males and 18-28 for females) & $59(18.85)$ & $24(30.8)$ & $35(14.9)$ & \\
\hline High BF $\%$ ( $\geq 21 \%$ for males and $\geq 29 \%$ for females) & $243(77.64)$ & $50(64.1)$ & $193(82.1)$ & \\
\hline
\end{tabular}

*Significant difference

Table 4. BMI and BF\%, according to gender stratified BMI groups (Mean $\pm S D$ )

\begin{tabular}{llll}
\hline Gender (n) & BMI Groups $(\boldsymbol{n})$ & BMI & BF \% \\
\hline \multirow{3}{*}{ Males (78) } & Underweight (12) & $17.49 \pm 0.72$ & $14.43 \pm 4.85$ \\
& Normal BMI (33) & $21.47 \pm 1.88$ & $18.38 \pm 4.88$ \\
F-Value & Overweight (20) & $26.69 \pm 1.24$ & $28.46 \pm 3.97$ \\
& Obese (13) & $35.11 \pm 6.21$ & $40.40 \pm 9.04$ \\
Females (235) & & $98.956^{* *}$ & $64.605^{* *}$ \\
& Underweight (26) & $17.27 \pm 0.92$ & $24.31 \pm 5.61$ \\
F-Value & Normal BMI (117) & $21.63 \pm 1.85$ & $32.75 \pm 6.85$ \\
\hline
\end{tabular}

\footnotetext{
** Significant differences for BMI groups at $\mathrm{p}<0.01$
} 
Table 5. BF\% in the study subjects according to their BMI group n (\%)

\begin{tabular}{|c|c|c|c|c|c|c|}
\hline \multirow[t]{2}{*}{ BMI group } & \multicolumn{2}{|c|}{$\begin{array}{l}\text { Low } \mathrm{BF} \% \text { ( } \leq 9 \text { for males and } \\
\leq 17 \text { for females) }\end{array}$} & \multicolumn{2}{|c|}{$\begin{array}{l}\text { Normal BF\% (10-20 for males and } \\
18-28 \text { for females) }\end{array}$} & \multicolumn{2}{|c|}{$\begin{array}{l}\text { High BF\% ( } \geq 21 \% \text { for males and } \\
\geq 29 \% \text { for females) }\end{array}$} \\
\hline & Males & Females & Males & Females & Males & Females \\
\hline Underweight & $2(16.7)$ & $3(11.5)$ & $9(75)$ & $18(69.2)$ & $1(8.3)$ & $5(19.20)$ \\
\hline Normal & $2(6.1)$ & $4(3.4)$ & $15(45.5)$ & $16(13.7)$ & $16(48.5)$ & 97 (82.9) \\
\hline Overweight & 0 & 0 & 0 & $1(1.7)$ & $20(100)$ & $59(98.3)$ \\
\hline Obese & 0 & 0 & 0 & 0 & $13(100)$ & $32(100)$ \\
\hline
\end{tabular}

Table 6. Dietary behaviors among the study population according to gender (\%)

\begin{tabular}{|c|c|c|c|c|c|}
\hline \multirow{2}{*}{ No } & \multirow{2}{*}{ Dietary Behavior } & \multirow{2}{*}{ Response } & \multicolumn{2}{|c|}{ Gender } & \multirow{2}{*}{ Chi- Square } \\
\hline & & & Male & Female & \\
\hline \multirow{4}{*}{ Q.1 } & \multirow{4}{*}{ Frequency of Breakfast } & Daily & 46.2 & 44.3 & 1.499 \\
\hline & & 2-3 times per week & 20.5 & 21.3 & $(p=0.683)$ \\
\hline & & $1-2$ times per week & 14.1 & 10.2 & \\
\hline & & Rarely or never & 19.2 & 24.3 & \\
\hline \multirow{4}{*}{ Q.2 } & \multirow{4}{*}{ Frequency of meals excluding snacks } & one meal Daily & 7.7 & 19.1 & 9.667 \\
\hline & & 2 Meals per day & 52.6 & 53.6 & $(\mathrm{p}=0.022 *)$ \\
\hline & & 3 Meals per day & 37.2 & 26.8 & \\
\hline & & 4 Meals per day & 2.6 & 0.4 & \\
\hline \multirow{4}{*}{ Q.3 } & \multirow{4}{*}{$\begin{array}{l}\text { Frequency of snacking apart from } \\
\text { regular meals }\end{array}$} & one time Daily & 30.8 & 36.2 & 1.068 \\
\hline & & 2 times per day & 34.6 & 33.6 & $(p=0.785)$ \\
\hline & & 3-4 times daily & 11.5 & 11.5 & \\
\hline & & Rarely or never & 23.1 & 18.7 & \\
\hline \multirow{4}{*}{ Q.4 } & \multirow{4}{*}{$\begin{array}{l}\text { Frequency of eating green, red, and } \\
\text { yellow colored vegetables }\end{array}$} & Daily & 15.4 & 12.0 & 3.551 \\
\hline & & 3-4 times per week & 16.7 & 13.7 & $(p=0.314)$ \\
\hline & & $1-2$ times per week & 37.2 & 31.6 & \\
\hline & & Rarely or never & 30.8 & 42.7 & \\
\hline \multirow{4}{*}{ Q.5 } & \multirow{4}{*}{ Frequency of eating fruits } & Daily & 6.4 & 5.1 & 0.468 \\
\hline & & 3-4 times per week & 12.8 & 14.5 & $(p=0.926)$ \\
\hline & & $1-2$ times per week & 35.9 & 33.3 & \\
\hline & & Rarely or never & 44.9 & 47.0 & \\
\hline \multirow{4}{*}{ Q.6 } & \multirow{4}{*}{ Frequency of eating fried foods } & Daily & 12.8 & 10.3 & 17.243 \\
\hline & & 3-4 times per week & 37.2 & 18.4 & $\left(\mathrm{p}=0.001^{*}\right)$ \\
\hline & & $1-2$ times per week & 33.3 & 33.8 & \\
\hline & & Rarely or never & 16.7 & 37.6 & \\
\hline & & Daily & 57.7 & 75.2 & 21.356 \\
\hline & & 3-4 times per week & 33.3 & 11.1 & $\left(\mathrm{p}=0.000^{*}\right)$ \\
\hline Q.7 & Frequency of eating with families & $1-2$ times per week & 7.7 & 9.8 & \\
\hline & & Rarely or never & 1.3 & 3.8 & \\
\hline & & Mainly meats & 9.0 & 9.1 & 9.539 \\
\hline 08 & What type of food do you think you & Mainly vegetables & 17.9 & 33.6 & $\left(\mathrm{p}=0.023^{*}\right)$ \\
\hline Q.8 & should eat to have a balanced nutrition & Meat, vegetables and other variety of foods & 62.8 & 44.0 & \\
\hline & & others & 10.3 & 13.4 & \\
\hline & & Rarely or never & 37.2 & 58.1 & 21.329 \\
\hline 0 & Frequency of consumption of carbonated & One to two times & 25.6 & 17.9 & $\left(\mathrm{p}=0.000^{*}\right)$ \\
\hline Q.9 & beverages per day & three times & 24.4 & 7.4 & \\
\hline & & four times and more & 12.8 & 16.6 & \\
\hline & & Rarely or never & 84.6 & 58.4 & 19.688 \\
\hline & Frequency of consumption of potato & One to two times & 7.7 & 16.9 & $\left(\mathrm{p}=0.000^{*}\right)$ \\
\hline Q.10 & chips per day & three times & 5.1 & 6.5 & \\
\hline & & four times and more & 2.6 & 18.2 & \\
\hline & & Rarely or never & 25.6 & 37.5 & 13.223 \\
\hline & Frequency of consumption of caffeinated & One to two times & 29.5 & 19.8 & $\left(\mathrm{p}=0.004^{*}\right)$ \\
\hline Q.11 & beverages per day & three times & 23.1 & 10.8 & \\
\hline & & four times and more & 21.8 & 31.9 & \\
\hline & & Rarely or never & 67.9 & 52.2 & 12.745 \\
\hline (1) & Frequency of consumption of sweets and & One to two times & 24.4 & 24.1 & $\left(\mathrm{p}=0.005^{*}\right)$ \\
\hline Q.12 & desserts per day & three times & 6.4 & 8.2 & \\
\hline & & four times and more & 1.3 & 15.5 & \\
\hline & & Rarely or never & 66.7 & 47.0 & 13.091 \\
\hline & Frequency of consumption of chocolates & One to two times & 16.7 & 23.3 & $\left(\mathrm{p}=0.004^{*}\right)$ \\
\hline Q.13 & per day & three times & 12.8 & 12.5 & \\
\hline & & four times and more & 3.8 & 17.2 & \\
\hline & & Rarely or never & 19.2 & 45.9 & 19.381 \\
\hline 14 & & One to two times & 24.4 & 20.2 & $\left(\mathrm{p}=0.000^{*}\right)$ \\
\hline Q.14 & Eatıng out in restaurants per week & three times & 25.6 & 12.9 & \\
\hline & & four times and more & 30.8 & 21.0 & \\
\hline
\end{tabular}

*Significant difference. 
Table 7. Dietary behaviors among the study population according to BMI groups (\%)

\begin{tabular}{|c|c|c|c|c|c|}
\hline No & Dietary Behavior & Response & $\begin{array}{l}\text { BMI Groups } \\
\text { Non-obese } \\
\text { (underweight and Normal BMI) }\end{array}$ & $\begin{array}{l}\text { Obese } \\
\text { (Overweight and Obese) }\end{array}$ & Chi- Square \\
\hline \multirow{4}{*}{ Q.1 } & \multirow{4}{*}{ Frequency of Breakfast } & Daily & 47.3 & 40.8 & 5.045 \\
\hline & & $2-3$ times per week & 22.9 & 18.4 & $(p=0.169)$ \\
\hline & & 1-2 times per week & 8.5 & 15.2 & \\
\hline & & Rarely or never & 21.3 & 25.6 & \\
\hline \multirow{4}{*}{ Q.2 } & \multirow{4}{*}{$\begin{array}{l}\text { Frequency of meals } \\
\text { excluding snacks }\end{array}$} & one meal Daily & 16.0 & 16.8 & 4.641 \\
\hline & & 2 Meals per day & 54.3 & 52.0 & $(\mathrm{p}=0.20)$ \\
\hline & & 3 Meals per day & 29.8 & 28.8 & \\
\hline & & 4 Meals per day & 0.0 & 2.4 & \\
\hline \multirow{4}{*}{ Q.3 } & \multirow{4}{*}{$\begin{array}{l}\text { Frequency of snacking } \\
\text { apart from regular meals }\end{array}$} & one time Daily & 37.2 & 31.2 & 7.280 \\
\hline & & 2 times per day & 36.2 & 30.4 & $(\mathrm{p}=0.05 *)$ \\
\hline & & 3-4 times daily & 11.7 & 11.2 & \\
\hline & & Rarely or never & 14.9 & 27.2 & \\
\hline \multirow{4}{*}{ Q.4 } & \multirow{4}{*}{$\begin{array}{l}\text { Frequency of eating green, } \\
\text { red, and yellow colored } \\
\text { vegetables }\end{array}$} & Daily & 12.2 & 13.7 & 2.219 \\
\hline & & 3-4 times per week & 13.3 & 16.1 & $(\mathrm{p}=0.528)$ \\
\hline & & 1-2 times per week & 36.2 & 28.2 & \\
\hline & & Rarely or never & 38.3 & 41.9 & \\
\hline \multirow{4}{*}{ Q.5 } & \multirow{4}{*}{ Frequency of eating fruits } & Daily & 4.8 & 6.5 & 2.469 \\
\hline & & 3-4 times per week & 15.4 & 12.1 & $(p=0.481)$ \\
\hline & & 1-2 times per week & 36.2 & 30.6 & \\
\hline & & Rarely or never & 43.6 & 50.8 & \\
\hline \multirow{4}{*}{ Q.6 } & \multirow{4}{*}{$\begin{array}{l}\text { Frequency of eating fried } \\
\text { foods }\end{array}$} & Daily & 9.6 & 12.8 & 2.050 \\
\hline & & 3-4 times per week & 24.6 & 20.8 & $(p=0.562)$ \\
\hline & & 1-2 times per week & 35.3 & 31.2 & \\
\hline & & Rarely or never & 30.5 & 35.2 & \\
\hline \multirow{4}{*}{ Q.7 } & \multirow{4}{*}{$\begin{array}{l}\text { Frequency of eating with } \\
\text { families }\end{array}$} & Daily & 72.7 & 68.0 & 5.499 \\
\hline & & 3-4 times per week & 18.2 & 14.4 & $(\mathrm{p}=0.142)$ \\
\hline & & 1-2 times per week & 6.4 & 13.6 & \\
\hline & & Rarely or never & 2.7 & 4.0 & \\
\hline \multirow{4}{*}{ Q.8 } & \multirow{4}{*}{$\begin{array}{l}\text { What type of food do you } \\
\text { think you should eat to } \\
\text { have a balanced nutrition }\end{array}$} & Mainly meats & 10.7 & 6.5 & 6.001 \\
\hline & & Mainly vegetables & 25.7 & 35.8 & $(\mathrm{p}=0.112)$ \\
\hline & & $\begin{array}{l}\text { Meat, vegetables and } \\
\text { other variety of foods }\end{array}$ & 52.4 & 43.1 & \\
\hline & & others & 11.2 & 14.6 & \\
\hline \multirow{4}{*}{ Q.9 } & \multirow{4}{*}{$\begin{array}{l}\text { Frequency of consumption } \\
\text { of carbonated beverages } \\
\text { per day }\end{array}$} & Rarely or never & 54.3 & 50.4 & 0.473 \\
\hline & & One to two times & 19.0 & 21.1 & $(\mathrm{p}=0.925)$ \\
\hline & & three times & 11.4 & 12.2 & \\
\hline & & four times and more & 15.2 & 16.3 & \\
\hline & & Rarely or never & 65.6 & 64.2 & 1.787 \\
\hline 10 & Frequency of consumption & One to two times & 15.1 & 13.8 & $(p=0.618)$ \\
\hline Q.10 & of potato chips per day & three times & 7.0 & 4.9 & \\
\hline & & four times and more & 12.4 & 17.1 & \\
\hline & & Rarely or never & 36.2 & 32.0 & 8.177 \\
\hline O 11 & Frequency of consumption & One to two times & 25.4 & 17.6 & $\left(\mathrm{p}=0.043^{*}\right)$ \\
\hline Q.11 & $\begin{array}{l}\text { of caffeinated beverages } \\
\text { per day }\end{array}$ & three times & 9.7 & 20.0 & \\
\hline & & four times and more & 28.6 & 30.4 & \\
\hline & & Rarely or never & 52.2 & 62.1 & 3.146 \\
\hline P & Frequency of consumption & One to two times & 26.3 & 21.0 & $(\mathrm{p}=0.370)$ \\
\hline Q.12 & $\begin{array}{l}\text { of sweets and desserts per } \\
\text { day }\end{array}$ & three times & 8.1 & 7.3 & \\
\hline & & four times and more & 13.4 & 9.7 & \\
\hline & & Rarely or never & 51.1 & 53.2 & 5.689 \\
\hline O 12 & Frequency of consumption & One to two times & 22.0 & 21.0 & $(\mathrm{p}=0.128)$ \\
\hline Q.13 & of chocolates per day & three times & 15.6 & 8.1 & \\
\hline & & four times and more & 11.3 & 17.7 & \\
\hline & & Rarely or never & 40.6 & 37.1 & 3.619 \\
\hline & Eating out in restaurants & One to two times & 18.2 & 25.8 & $(p=0.306)$ \\
\hline Q.14 & per week & three times & 18.2 & 12.9 & \\
\hline & & four times and more & 23.0 & 24.2 & \\
\hline
\end{tabular}


Table 8. Dietary behaviors among the study population according to BF\% groups (\%)

\begin{tabular}{|c|c|c|c|c|c|}
\hline No & Dietary Behavior & Response & $\begin{array}{l}\text { BF\% Groups } \\
\text { Non-obese (Low and Normal BF\%) }\end{array}$ & Obese (High BF\% ) & Chi- Square \\
\hline \multirow{4}{*}{ Q.1 } & \multirow{4}{*}{ Frequency of Breakfast } & Daily & 58.6 & 40.7 & 12.353 \\
\hline & & 2-3 times per week & 7.1 & 25.1 & $\left(\mathrm{p}=0.006^{*}\right)$ \\
\hline & & $1-2$ times per week & 10.0 & 11.5 & \\
\hline & & Rarely or never & 24.3 & 22.6 & \\
\hline \multirow{4}{*}{ Q.2 } & \multirow{4}{*}{$\begin{array}{l}\text { Frequency of meals } \\
\text { excluding snacks }\end{array}$} & one meal Daily & 15.7 & 16.5 & 4.201 \\
\hline & & 2 Meals per day & 44.3 & 56.0 & $(p=0.241)$ \\
\hline & & 3 Meals per day & 38.6 & 26.7 & \\
\hline & & 4 Meals per day & 1.4 & 0.8 & \\
\hline \multirow{4}{*}{ Q.3 } & \multirow{4}{*}{$\begin{array}{l}\text { Frequency of snacking } \\
\text { apart from regular } \\
\text { meals }\end{array}$} & one time Daily & 32.9 & 35.4 & 2.661 \\
\hline & & 2 times per day & 28.6 & 35.4 & $(p=0.447)$ \\
\hline & & 3-4 times daily & 15.7 & 10.3 & \\
\hline & & Rarely or never & 22.9 & 18.9 & \\
\hline \multirow{4}{*}{ Q.4 } & \multirow{4}{*}{$\begin{array}{l}\text { Frequency of eating } \\
\text { green, red, and yellow } \\
\text { colored vegetables }\end{array}$} & Daily & 12.9 & 12.8 & 5.376 \\
\hline & & 3-4 times per week & 22.9 & 12.0 & $(p=0.146)$ \\
\hline & & 1-2 times per week & 30.0 & 33.9 & \\
\hline & & Rarely or never & 34.3 & 41.3 & \\
\hline \multirow{4}{*}{ Q.5 } & \multirow{4}{*}{$\begin{array}{l}\text { Frequency of eating } \\
\text { fruits }\end{array}$} & Daily & 8.6 & 4.5 & 10.934 \\
\hline & & 3-4 times per week & 20.0 & 12.4 & $\left(\mathrm{p}=0.012^{*}\right)$ \\
\hline & & 1-2 times per week & 18.6 & 38.4 & \\
\hline & & Rarely or never & 52.9 & 44.6 & \\
\hline \multirow{4}{*}{ Q.6 } & \multirow{4}{*}{$\begin{array}{l}\text { Frequency of eating } \\
\text { fried foods }\end{array}$} & Daily & 12.9 & 10.3 & 4.725 \\
\hline & & 3-4 times per week & 27.1 & 21.9 & $(p=0.193)$ \\
\hline & & 1-2 times per week & 22.9 & 36.8 & \\
\hline & & Rarely or never & 37.1 & 31.0 & \\
\hline \multirow{4}{*}{ Q.7 } & \multirow{4}{*}{$\begin{array}{l}\text { Frequency of eating } \\
\text { with families }\end{array}$} & Daily & 80.0 & 68.2 & 3.990 \\
\hline & & 3-4 times per week & 12.9 & 17.8 & $(p=0.263)$ \\
\hline & & 1-2 times per week & 5.7 & 10.3 & \\
\hline & & Rarely or never & 1.4 & 3.7 & \\
\hline \multirow{5}{*}{ Q.8 } & \multirow{5}{*}{$\begin{array}{l}\text { What type of food do } \\
\text { you think you should } \\
\text { eat to have a balanced } \\
\text { nutrition }\end{array}$} & Mainly meats & 5.7 & 10.0 & 2.497 \\
\hline & & Mainly vegetables & 25.7 & 30.8 & $(p=0.476)$ \\
\hline & & Meat, vegetables & & & \\
\hline & & $\begin{array}{l}\text { and other variety of } \\
\text { foods }\end{array}$ & 55.7 & 46.7 & \\
\hline & & others & 12.9 & 12.5 & \\
\hline \multirow{4}{*}{ Q.9 } & & Rarely or never & 50.7 & 53.4 & 2.074 \\
\hline & consumption of & One to two times & 23.2 & 18.9 & $(p=0.557)$ \\
\hline & carbonated beverages & three times & 14.5 & 10.9 & \\
\hline & & four times and more & 11.6 & 16.8 & \\
\hline & & Rarely or never & 72.9 & 62.8 & 2.629 \\
\hline 010 & $\begin{array}{l}\text { Frequency of } \\
\text { sonsumntion }\end{array}$ & One to two times & 11.4 & 15.5 & $(p=0.452)$ \\
\hline Q.10 & $\begin{array}{l}\text { consumption or potato } \\
\text { chins ner day }\end{array}$ & three times & 5.7 & 6.3 & \\
\hline & & four times and more & 10.0 & 15.5 & \\
\hline & Frequency of & Rarely or never & 32.9 & 35.0 & 0.244 \\
\hline Q.11 & consumption of & One to two times & 21.4 & 22.5 & $(\mathrm{p}=0.970)$ \\
\hline Q.11 & caffeinated beverages & three times & 14.3 & 13.8 & \\
\hline & per day & four times and more & 31.4 & 28.8 & \\
\hline & & Rarely or never & 57.1 & 55.8 & 0.763 \\
\hline 0.12 & $\begin{array}{l}\text { Frequency of } \\
\text { consumption of sweets }\end{array}$ & One to two times & 21.4 & 25.0 & $(\mathrm{p}=0.858)$ \\
\hline र.12 & $\begin{array}{l}\text { consumption or sweets } \\
\text { and desserts per day }\end{array}$ & three times & 7.1 & 7.9 & \\
\hline & & four times and more & 14.3 & 11.2 & \\
\hline & & Rarely or never & 58.0 & 50.2 & 2.037 \\
\hline & Frequency of & One to two times & 15.9 & 23.2 & $(\mathrm{p}=0.565)$ \\
\hline Q.13 & consumption of & three times & 11.6 & 12.9 & \\
\hline & chocolates per day & four times and more & 14.5 & 13.7 & \\
\hline & & Rarely or never & 44.3 & 37.8 & 9.023 \\
\hline & Eating out in & One to two times & 8.6 & 24.9 & $\left(\mathrm{p}=0.027^{*}\right)$ \\
\hline Q.14 & restaurants per week & three times & 21.4 & 14.5 & \\
\hline & & four times and more & 25.7 & 22.8 & \\
\hline
\end{tabular}

*Significant difference

Collectively, $40 \%$ of the study subjects were overweight and obese, with $25.6 \%$ of the total subjects being overweight (BMI 25- 29.99) while $14.4 \%$ were obese
(BMI $\geq 30$ ) (Table 3). According to BMI gender stratification, higher prevalence of underweight $(15.4 \%$ vs. $11.1 \%)$ and obesity (16.7\% vs. $13.6 \%)$ in males was observed as 
compared to females. The differences were not statistically significant $(\chi 2=1.963, \mathrm{P}=0.580)$. On the other hand, there was a significant difference in the prevalence of high risk WHR between males (32.1\%) and females (54.9\%); with females having 2.5 times increased risk of having high risk WHR as compared to males $(\chi 2=12.226, \quad \mathrm{P}=0.000$; $\mathrm{OR}=2.580$ (95\% CI: 1.503-4.430). Similarly, prevalence of high $\mathrm{BF} \%$ exhibited significant differences between males and females $(\chi 2=11.051, \mathrm{P}=0.004)$, with females being at higher risk for high $\mathrm{BF} \%$ as compared to males.

Table 4 presents mean $\mathrm{BMI}$ and $\mathrm{BF} \%$, across $\mathrm{BMI}$ groups, which were significantly lowest $(\mathrm{P}<0.01)$ for underweight and highest for obese group for both genders. However Table 5 signifies that body composition analysis assures better recognition of body fat content than BMI alone, and also suggests that it is imperative to consider gender disparity too. It is evident from this table that the distribution of $\mathrm{BF} \%$ varies across genders for underweight and normal BMI groups. In male underweight BMI group nearly $75 \%$ have normal $\mathrm{BF} \%$ and $8.3 \%$ have high $\mathrm{BF} \%$; whereas female underweight BMI group have only $69.3 \%$ with normal $\mathrm{BF} \%$ and around $19.2 \%$ have high BF\%. Similarly, male normal BMI group have $45.5 \%$ with normal $\mathrm{BF} \%$ and $48.5 \%$ with high $\mathrm{BF} \%$; while in female normal BMI group only $13.7 \%$ have normal $\mathrm{BF} \%$ while nearly $82.9 \%$ have high $\mathrm{BF} \%$. Both male and female overweight and obese groups have 100 percent high $\mathrm{BF} \%$.

Table 6 presents dietary behaviors among the study population and according to gender of the subjects. One interesting finding of the current study is that only $44 \%$ of female students believed in consumption of variety of foods as a key factor in achieving balanced diet, when compared with $62.8 \%$ of male students with the same belief $(\mathrm{x} 2=$ 9.667; $\mathrm{P}=0.022$ ). Overall, 24.3 and $19.1 \%$ of female students were at risk for skipping breakfast and meals respectively. High prevalence of rare or never vegetable and fruit consumption patterns (males 30.8 and $44.9 \%$ and females 42.7 and $47 \%$, respectively) also suggest the need for awareness of health promoting dietary habits among the study population.

Gender differences were also found towards health risk food preferences. As compared to females, males reported significantly higher consumption of fried foods $(\mathrm{x} 2=17.243$; $\mathrm{P}=0.001)$; carbonated beverages $(\mathrm{x} 2=21.329 ; \mathrm{P}=0.000)$ and fast foods $(\mathrm{x} 2=19.381 ; \mathrm{P}=0.000)$. As compared to males, females reported significantly higher consumption of potato chips $(\mathrm{x} 2=19.688 ; \mathrm{P}=0.000)$; caffeinated beverages $(\mathrm{x} 2=$ 13.223; $\mathrm{P}=0.004) ;$ sweets and desserts $(\mathrm{x} 2=12.745$; $\mathrm{P}=0.005)$; and chocolates $(\mathrm{x} 2=13.091 ; \mathrm{P}=0.004)$.

Table 7 presents dietary habits of BMI groups (obese and non obese) among the study population. Overall, obese BMI group (overweight and obese) appears to be at risk for health risk dietary habits like skipping breakfast, low consumption of vegetables and fruits, eating fried foods, potato chips, caffeinated beverages, chocolates as compared to non-obese (normal and underweight) BMI group. However the trend differences were not statistically significant except for consumption of caffeinated beverages ( $\mathrm{x} 2=8.177 ; \mathrm{P}=0.043)$. Snacking was significantly inversely associated with BMI $(\mathrm{x} 2=7.280 ; \mathrm{P}=0.05)$ indicating positive role in the prevention of obesity. Only $43.1 \%$ of overweight and obese BMI group believed in variety as the basis of balanced diet as compared to $52.4 \%$ of normal and underweight BMI group.

A similar trend was observed for BF\% groups (Table 8). Skipping breakfast was significantly associated with high $\mathrm{BF} \%$ prevalence $(\mathrm{x} 2=12.353 ; \mathrm{P}=0.006)$. Other health risk dietary habits significantly associated for high $\mathrm{BF} \%$ include low consumption of fruits $(\mathrm{x} 2=10.934 ; \mathrm{P}=0.012)$, and fast food consumption ( $\mathrm{x} 2=9.203 ; \mathrm{P}=0.027$ ) as compared to low and normal $\mathrm{BF} \%$ groups. Only $46.7 \%$ of high $\mathrm{BF} \%$ group believed in variety as the basis of balanced diet as compared to $55.7 \%$ of low and normal $\mathrm{BF} \%$ groups.

\section{Discussion}

Rising epidemic of obesity and consequent increase in obesity related chronic diseases is currently the major public health concern threatening healthcare systems, economies and individual lives. However, easy solutions are unlikely given the complex interactions of lifestyle factors in the etiology of the obesity. Prevention, especially from younger ages onwards, is always the effective strategy to combat the consequences of obesity in communities. Lifestyle changes across the world primarily in dietary habits and physical activity patterns were linked with this epidemic of obesity. Identifying the poor dietary choices and other health risk behaviors early in life along with multifaceted efforts to improve right choices will therefore go a long way in turning around the epidemic.

The present study indicates the prevalence of overweight and obesity in the university students as $25.6 \%$ and $14.4 \%$. These results are in acceptance with previous studies done on university students from Saudi Arabia [7, 8, 14 and 15]. Another recent study on Saudi female university students reported an overweight of $31.4 \%$ and obesity of $16.5 \%$ [16]. A recent review [2] attempted to comprehend the changes in prevalence trends of obesity in the Gulf region over a period of time indicated that in Saudi Arabia, obesity (BMI $\geq 30$ ] among young women was consistently higher then young men in 1990's although the gap narrowed over time by 2000's. However for overweight the prevalence rates were same for both genders across the time according to the studies mentioned in this review. In the current study also, overweight prevalence rates were same for young men and women.

Recent literature indicates that body composition analysis is more desirable determinant of obesity rather than BMI alone [17]. The present study results were also in support of this assumption. Percent body fat distribution varies within the underweight and normal BMI groups demonstrating complex distribution of the adiposity in these groups and these results correlate with other studies from the region [8]. As suggested by Meeuwsen et al. [18] these results also 
confirm the striking finding that BMI can predict BF\% fairly well among both genders among the overweight and obese BMI groups. It is the variation among those with underweight and normal BMI which is more poorly associated with $\mathrm{BF} \%$ that is of greater concern. In near future, it is unlikely for BMI to be replaced by any other measures of fatness for large epidemiological studies since it can be measured effortlessly. However, caution is vital when applying the BMI concept for underweight and normal BMI population groups given that variation in $\mathrm{BF} \%$ was poorly detected by BMI in these groups.

The Kingdom's National Nutrition Survey indicated the changing dietary preferences of the Saudi population towards eating non-nutritional and high calorie snacks like fried foods and carbonated drinks commonly in day to day life [5]. These changes reflect the shifting socio-environmental conditions which can predispose young adults to obesity. Only limited research is available from KSA region which focused on dietary and other lifestyle behavior patterns among young adults [7, 8]. Few other studies address about lifestyle trends in general population $[5,19,20]$. All the studies raise alarm for increasing sedentary lifestyle and fast food culture.

The current study documents gender specific differences for health risk and health promoting dietary behaviors. Obese males spend less time with families, eat more fried foods and fast foods and drink more carbonated beverages as compared to obese females who prefer snacking on potato chips, chocolates, cakes, sweets and would prefer to drink more caffeinated drinks. Both genders were at risk for dietary behaviors like eating less fruits and vegetables.

Interestingly, in this study gender differences (52.4 and $43.1 \%$ for males and females, respectively) were also noted for belief in variety of the diet as key for balanced nutrition. A study done on male college students from Qassim area in KSA which is proximally closer to Hail also reported similar results that only $59.7 \%$ of subjects agreed to variety as key for balanced nutrition. These figures were alarmingly low as compared to a Lebanese study which reported 77 and $73 \%$ respectively [13] and an American study which reported $94.4 \%$ of the students agreed eating variety of foods as vital for good health [22].

Breakfast skipping behavior, eating less fruits and eating frequently in restaurants have established significant association with high BF\% in the present study. Gender wise analysis (data not presented) also confirmed the inverse association between low consumption of fruits with high $\mathrm{BF} \%$. These findings are interesting particularly the association of habit of low consumption of fruits with high $\mathrm{BF} \%$ given the fact many studies [8, 16, 23, 24] have indicated the prevalence of habitual low consumption of fruits and vegetables in Saudi Arabia.

Findings of the current study also suggest habit of frequent snacking behavior inverse association with BMI. A recent study [8] which was done on male Saudi college students also indicated similar relationship for snacking with BMI proving Bellisle et al.[25] assumption, i.e. "nibbling meal pattern" helps in avoidance of obesity rather than the "gorging meal pattern".

The study results on dietary habits help in identifying the crucial missing links in understanding epidemic of obesity which in turn helps in tailor made nutritional interventions to promote healthy behaviors among younger generations. This study which was done simultaneously on both genders from the same population gains huge importance in understanding gender disparity in health risk dietary behaviors, given the fact that only one study is available addressing obesity and dietary behaviors association among only male KSA young adult population [8]. This study results therefore emphasizes the need for future research focusing on developing differential primary prevention intervention strategies catering to varying gender requirements.

The current study encompasses several limitations. The findings of our study could be limited since it was a cross-sectional study which may not be sufficient enough to evaluate dietary behaviors of the study population. Also majority students who participated were from science colleges and therefore may not be representing the entire university colleges. The study focused primarily on dietary behaviors association with $\mathrm{BMI}$ and $\mathrm{BF} \%$ which may limit our understanding of other lifestyle factors which are known to confound them.

\section{Conclusion}

In summary, our findings were consistent with previous studies indicating a high prevalence of overweight and obesity defined on the basis of both BMI as well as body composition analysis among university students in KSA. The contributing factors for obesity were different for males and females. Health risk dietary behaviors like, low vegetables and fruits intake, high consumption of fast foods, fried foods, carbonated and caffeinated beverages coupled with inadequate physical activity were most common risk factors for obesity among the participants. The study findings emphasize the need for planning gender-specific nutrition education programs to reverse and reduce the epidemic of obesity and safeguard the future health of young people and adults in Saudi Arabia.

Universities provide the last opportunity to reach large number of students before they enter realms of life for behaviour modification. Given the medical care costs and complexities in living with chronic diseases it is better to prevent obesity early in the life so as to promote healthy nations. The study findings therefore will have several important implications for future prevention efforts.

\section{Acknowledgements}

A special note of appreciation goes for the fourth year students of the Department of Clinical Nutrition/College of Applied Medical Sciences at $\mathrm{UOH}$ for their assistance in conducting the research, and for the students of $\mathrm{UOH}$ for their cooperation, and to all UOH staff who helped in this study. 


\section{References}

[1] The United States, Census Bureau, International Data Base. http://www.census.gov/population/international/data/idb/cou ntry.php. Accessed on 16th Nov 2013.

[2] Ng SW, Zaghloul S, Ali HI, Harrison G, Popkin BM,“The prevalence and trends of overweight, obesity and nutrition-related non-communicable diseases in the Arabian Gulf States", Obes Rev., Vol 12, 2011, pp. 1-13.

[3] Musaiger AO, Al-Hazzaa HM, Takruri HR, Mokhatar N, "Change in nutrition and lifestyle in the Eastern Mediterranean region: health impact", J NutrMetab., Vol2012, 2012, 436762, doi: 10.1155/2012/436762

[4] Al-Othaimeen AI, Al-Nozha M, Osman AK, “Obesity: an emerging problem in Saudi Arabia. Analysis of data from the National Nutrition Survey", East Mediterr Health J., Vol 13, 2007, pp. 441-48.

[5] Al-Nozha MM, Al-Mazrou YY, Al-Maatouq MA, Arafah MR , Khalil MZ, Khan NB et al,"Obesity in Saudi Arabia”, Saudi Med J., Vol 26, 2005, pp. 824-9.

[6] El-Hazmi MA, Warsy AS, "A comparative study of prevalence of overweight and obesity in children in different provinces of Saudi Arabia", J Tropic Pediatr.,Vol 48, 2002, pp. 172-7.

[7] Rasheed P, Abou-Hozaifa BM, Kahn A,“Obesity among young Saudi female adults: a prevalence study on medical and nursing students", Public Health., Vol 108, 1994, pp. 289-94.

[8] Al-Rethaia AS, Fahmy AE, Al-Shwaiyat NM, "Obesity and eating habits among college students in Saudi Arabia: a cross sectional study",Nutr J., Vol 9, 2010, pp. 1-10.

[9] Langley-Evans S, "Nutrition: A Lifespan Approach: Nutrition and Adolescence", 3rd ed., Wiley-Blackwell Publishers, 2009, pp. 170.

[10] "Clinical guidelines on the identification, evaluation, and treatment of overweight and obesity in adults-the evidence report", National Institutes of Health Obesity Research, Supp. 2, 1998, pp. 51S-209S.

[11] "Waist Circumference and Waist-Hip Ratio. Report of a WHO Expert Consultation", Geneva, 8-11 Dec, 2008. http://whqlibdoc.who.int/publications/2011/9789241501491 _eng.pdf Accessed on 18th, Nov, 2013.

[12] "The Precision Body Composition Analyzer InBody720 User's Manual", http://www.imr-switzerland.org/downloads/in-body-720-ma nual.pdf Accessed on 18th, Jan, 2012.

[13] Yahia N, Achkar A, Abdallah A, Rizk S,"Eating habits and obesity among Lebanese university students",Nutr J., Vol 30, 2008, pp. 7-32.

[14] Al Turki YA, "Overweight and obesity among university students, Riyadh, Saudi Arabia.", Middle East J Family Med.,Vol 2(5) March, 2007. Accessed on 21st Nov, 2013. http://www.mejfm.com/journal/March2007/Overweight.htm

[15] AbolfotouhAM, Al-Alwan AIA, Al-Rowaily MA,"Prevalence of metabolic abnormalities and association with obesity among Saudi college students", Intern J Hypertens., Vol2012, 2012, 819726. doi: 10.1155/2012/819726.

[16] Al Qauhiz NM,“Obesity among Saudi female university students: dietary habits and health behaviors", J Egypt Public Health Assoc., Vol 85, 2010, pp. 45-59.

[17] Frankenfield DC, Rowe WA, Cooney RN, Smith JS, Becker $\mathrm{D}$, "Limits of body mass index to detect obesity and predict body composition", Nutrition, Vol 17, 2001, pp. 26-30.

[18] Meeuwsen S, Horgan GW, Elia M, "The relationship between BMI and percent body fat, measured by bioelectrical impedance, in a large adult sample is curvilinear and influenced by age and sex",ClinNutr., Vol 29, 2010, pp. 560-6.

[19] Al-Hazzaa H, "The public health burden of physical inactivity in Saudi Arabia”, J Fam Community Med., Vol 11, 2004, pp. 45-51.

[20] Al-Nuaimr AA, Al-NakeebY, Lyons M, Al-Hazzaa HA, Nevill A, Collins P,et al., "The prevalence of physical activity and sedentary behaviors relative to obesity among adolescents from Al-Ahsa, Saudi Arabia: rural versus urban variations", J NutrMetab., Vol2012, 2012, 417589, doi:10.1155/2012/417589.

[21] Berger G, Peerson A, "Giving young Emirati women a voice: participatory action research on physical activity", Health Place., Vol 15, 2009, pp. 117-24.

[22] Davy SR, Benes BA, Driskell JA, “Sex differences in dieting trends, eating habits, and nutrition beliefs of a group of Midwestern college students", J Am Diet Assoc., Vol 106, 2006 pp. 1673-7.

[23] Washi SW, Ageib MB, "Poor diet quality and food habits are related to impaired nutritional status in 13- to 18-year-old adolescents in Jeddah",Nutr Res., Vol 30, 2010, pp. 527-34.

[24] Amin TT, Al-Sultan AI, Ali A,“Overweight and obesity and their relation to dietary habits and socio-demographic characteristics among male primary school children in Al-Hassa, Kingdom of Saudi Arabia",Eur J Nutr.,Vol 47, 2008, pp. 310-8.

[25] Bellisle F, McDevitt R, Prentice AM,"Meal frequency and energy balance”, Br J Nutr., Vol 77, 1997, pp. S57-S70. 